\title{
Computational studies of granular mixing
}

\author{
Joseph J. McCarthy ${ }^{\mathrm{a}, *}$, Devang V. Khakhar ${ }^{\mathrm{b}}$, Julio M. Ottino ${ }^{\mathrm{c}}$ \\ ${ }^{a}$ Department of Chemical and Petroleum Engineering, University of Pittsburgh, 1249 Benedum Hall, Pittsburgh, PA 15261, USA \\ ${ }^{\mathrm{b}}$ Department of Chemical Engineering, Indian Institute of Technology-Bombay, Powai, Bombay 400076, India \\ ${ }^{\mathrm{c}}$ Department of Chemical Engineering, Northwestern University, Evanston, IL 60208, USA
}

\begin{abstract}
Particulate systems have proven difficult to probe experimentally in many instances. Simulations of granular flows, and mixing flows in particular, provide a useful means of studying particulate behavior. Mixing flows generate large scale patterns and structures which can be easily visualized. Thus, mixing studies provide a means of indirectly examining granular flows. In this paper we review recent computational studies of tumbler mixing, focusing on two very different, yet complementary, techniques: Particle Dynamics and Lagrangian Simulation. We discuss mixing in different tumbler geometries, as well as segregation and cohesive effects.
\end{abstract}

Keywords: Granular mixing; Particle Dynamics; Lagrangian Simulation

\section{Introduction}

Why focus on mixing? Unarguably, the first part of the answer has to do with the practical implications of the results. A second aspect is somewhat less obvious. Mixing flows generate large scale patterns and structures which can be easily visualized. Thus, mixing studies provide a means of indirectly studying granular flows which would otherwise be hard to probe experimentally. In this paper, we explore two complementary methods of simulation of particulate materials in the context of granular mixing: Lagrangian Simulations (LS) and Particle Dynamics (PD) methods. Simulations and experiments of mixing and segregation in a prototype system - a rotating drum - are considered here. The role of cohesion due to liquid bridging in mixing is also briefly discussed. Fig. 1 shows a schematic outline or road map of the paper's contents.

A fundamental understanding of mixing of particulate systems is lacking, despite its potentially beneficial impact on a number of industries, such as pharmaceuticals, metallurgy, and ceramics, to name but a few. Possibly the biggest hindrance is that there is no accepted set of universal governing equations describing granular flow. This situation can be attributed not only to the intrinsic physical complexity of the problems, but also, in part, to the difficulty in experimentally measuring the bulk properties of the material (e.g., stress, strain, voidage, etc.). While there have been notable advances in non-invasive experimental methods (positron tomography [1], nuclear magnetic resonance [2], and gamma ray tomography [3]), which circumvent problems associated with bed opacity, computational modeling of particulate flows has gained widespread acceptance as a viable alternative or supplement to experiments. One particularly fruitful use of computational modeling is for the study of mixing flows.

This paper is organized as follows: In Section 2, we briefly discuss the application of continuum modeling techniques to LS of mixing problems. We pay particular attention to the application of this technique to mixing in a tumbler. In Section 3, we discuss the utility of "model" PD Simulations and discuss how this approach may be used to complement the LS. Finally, we conclude with a brief discussion of the potential future significance of this type of approach.

\section{Lagrangian Simulations}

Continuum mechanics-inspired studies of rapid flows - characterized by fluid-like flow of granular materials 


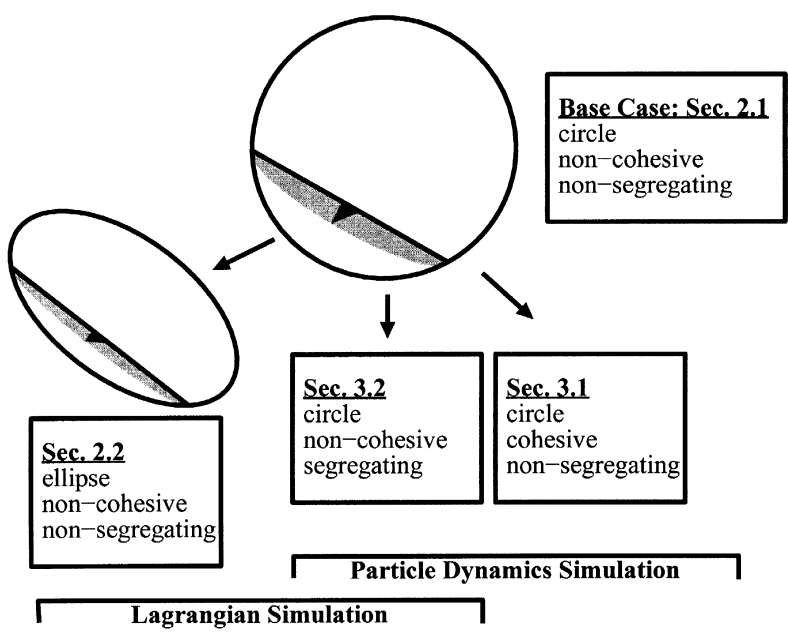

Fig. 1. Road map and organization of the material covered in this paper. While the flow in a tumbler exhibits differing flow regimes depending on rotation rate, here we focus exclusively on the rolling regime.

- have been shown to give good predictions for shear flows of nearly elastic spherical particles. Despite their success in simple flows, extensions of this approach to more complex flows is difficult. However, for certain classes of complex flows, which can be decomposed into distinct regions of uniform simple flow, satisfactory results for flow modeling can be obtained from a continuum analysis. LS, based on such continuum models, then provide a useful means of probing mixing. One example of such a flow is a rotated tumbler operated in the rolling regime. In this flow, there exists a rapid flow region at the free surface (the cascading layer), and a fixed bed region which undergoes solid body rotation. A nearly one-dimensional continuum-based description of the flow in the layer, coupled with the solid-body rotation within the bed, generates quantitative predictions of global mixing rates and concentration profiles in both segregating and nonsegregating flows.

\subsection{Mixing in a cylindrical tumbler}

In the rolling regime, rotation of a tumbler results in a flow of particles from the fixed bed into the cascading layer in the uphill half of the surface and a flow from the layer into the fixed bed in the downhill half (see Fig. 2). In order to carry out LS of the particle motion within a rotating tumbler, the velocity and layer thickness profiles for the flowing layer must be obtained. To that end, we use a macroscopic balance approach to obtain the velocity and the layer thickness, which agree with previous experimental findings (see Ref. [2]). A detailed discussion of the macroscopic balance approach has been reported elsewhere (see Ref. [4]) and only the results necessary for LS will be discussed below (note that, unless specified other- wise, all equations are made dimensionless with the halflength of the free surface, $L$, and the rotation rate, $\omega$ ).

Within the layer, the components of the velocity at any point, $v_{x}$ and $v_{y}$, respectively, are given by:

$v_{x}=\frac{\mathrm{d} x}{\mathrm{~d} t}=2 u\left(1+\frac{y}{\delta(x)}\right)$,

$v_{y}=\frac{\mathrm{d} y}{\mathrm{~d} t}=-x\left(\frac{y}{\delta(x)}\right)^{2}$,

where $y$ is the coordinate perpendicular to the free surface (and pointing out of the bed), $x$ is the coordinate parallel to the free surface, $\delta(x)$ is the layer thickness, $t$ is time, and $u$ is the average velocity within the layer (which is roughly constant).

Additionally, in the general case, we must consider whether collisions within the layer will contribute significantly to the particle motion. In order to examine this issue in a LS, we must augment the above-equations with a diffusive term in the expression for $v_{y}$ (as $v_{x}$ is much larger than $v_{y}$, we assume that particle diffusion in the $x$ direction is very small, relative to advection). The new $v_{y}$, now applicable to situations with finite diffusive mixing, is given by:

$v_{y}=\frac{\mathrm{d} y}{\mathrm{~d} t}=-x\left(\frac{y}{\delta(x)}\right)^{2}+S(t)$,

where $\Delta y=\int_{t}^{t+\Delta t} S(t) \mathrm{d} t$ (the "jump" in $y$ position per time step) is a Gaussian random number with variance $2 D \Delta t$ and $D$ is the effective diffusivity.

Using this model, a typical particle trajectory within the tumbler is comprised of a sequence of rotational and translational steps. If the particle is in the bed, $y<\delta(x)$, it is rotated as a solid body until it enters the layer, $y>\delta(x)$, whereupon it is advected according to Eqs. (1) and (3). By varying the effective diffusivity, the relative importance of

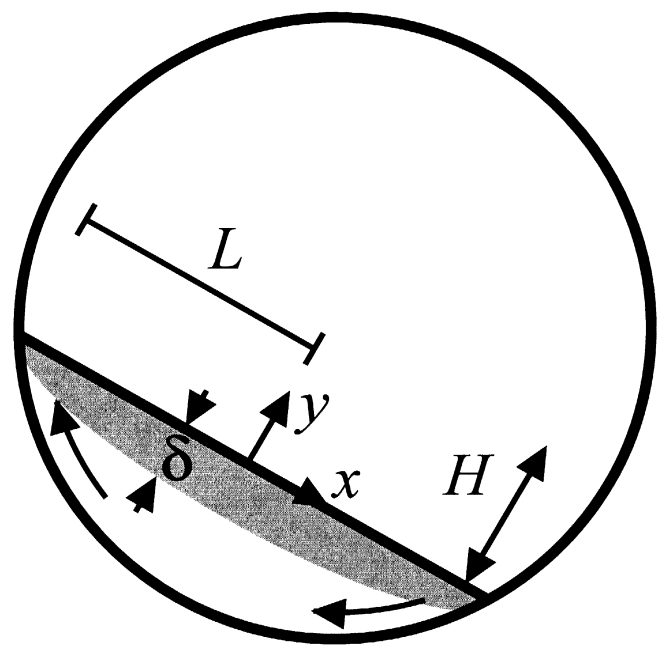

Fig. 2. Schematic representation of a cylindrical tumbler operated in the rolling regime. 


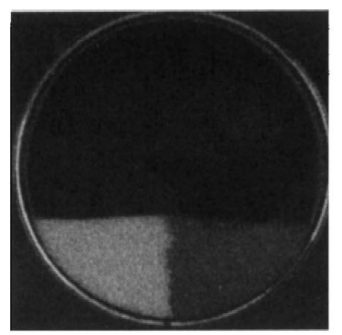

Initial condition

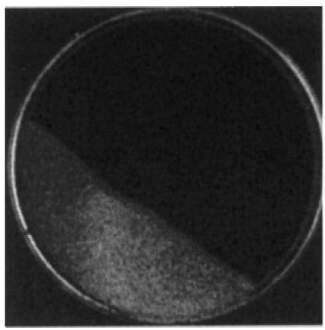

1.2 Revolutions

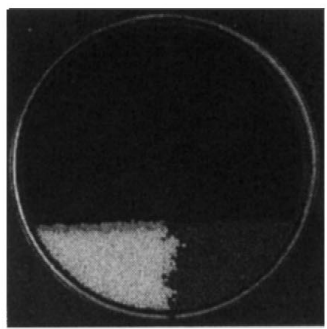

Initial condition

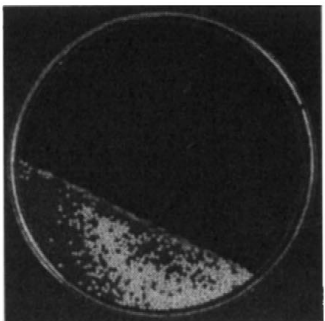

b)

0.7 Revolutions

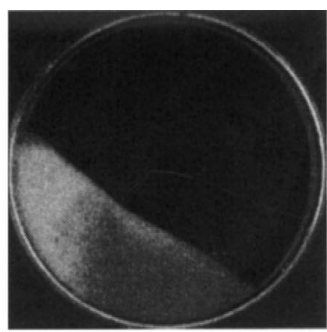

0.5 Revolutions

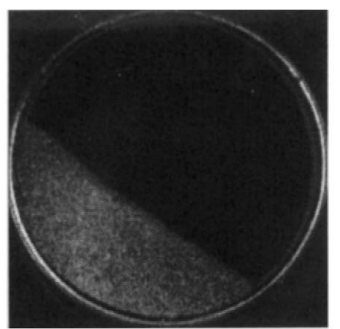

1.7 Revolutions

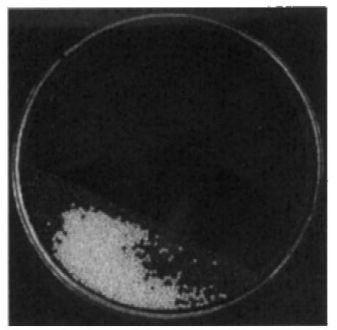

0.4 Revolutions

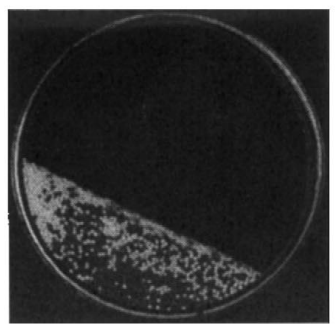

1 Revolutions
Fig. 3. Experimental time evolution of the mixing process for two differing materials. (a) Sugar crystals (SC) filled to $H=0.35$. (b) Sugar balls (SB) filled to $H=0.42$. Reprinted with permission from Khakhar et al. [4]. Copyright 1997 American Institute of Physics.

advective and diffusive components of the mixing can be compared (this idea is explored in more detail in the next section).

In the LS, particles initially have a specified distribution (here segregated right and left). They are advected according to the procedure described above, and then the mixing of the particles is analyzed. For a quantitative comparison of the rates of mixing, the intensity of segregation, $I_{\mathrm{s}}$, is calculated from:

$I_{\mathrm{s}}=\left(\frac{\sum_{i=1}^{N}\left(\phi_{i}-\phi_{m}\right)^{2}}{N-1}\right)^{1 / 2}$ where $\phi_{i}$ are the concentrations at a set of $N$ uniformly distributed points in the bed and $\phi_{m}$ is the overall concentration [5]. The point concentration is calculated as the fraction of one type of particle in a box of a specified size centered on the point. This data is then compared to experiment to verify the simulation.

In the mixing experiments, identical particles of different colors are initially placed in two halves of a short cylinder using a separator. The separator is removed, the cylinder is mounted on the rollers and rotated, and digital images are recorded at short time intervals as the mixing proceeds. The images are analyzed by thresholding of the intensities to identify each type of particle. The local concentrations and the intensity of segregation are then calculated. Fig. 3 shows the time evolution of the mixed state for two mixing experiments; the variation in the intensity of segregation is shown in Fig. 4 with lines fitted to the initial decay of the intensity of segregation.

In order to mimic the experimental flow, several parameters are needed for the LS: the filling parameter, $H$; the maximum layer thickness, $\delta_{0}$; and the effective diffusivity, $D$ (note that the average velocity, $u$, is obtained from a mass balance once the other parameters are known). The filling parameter and the maximum layer thickness can both be easily measured via inspection, however, the effective diffusivity must be estimated. A qualitative comparison of an experimental snapshot to computations with differing values of $D$ shows reasonably good agreement at $D=3 \times 10^{-3}$ for the sugar balls (see Fig. 5). Similarly, a quantitative comparison of the change in the intensity of segregation with time also yields a value of $D=3 \times 10^{-3}$ (see Fig. 6). Using either of these methods, the estimated diffusion coefficient is consistent with our experimental measurements at any filling level.

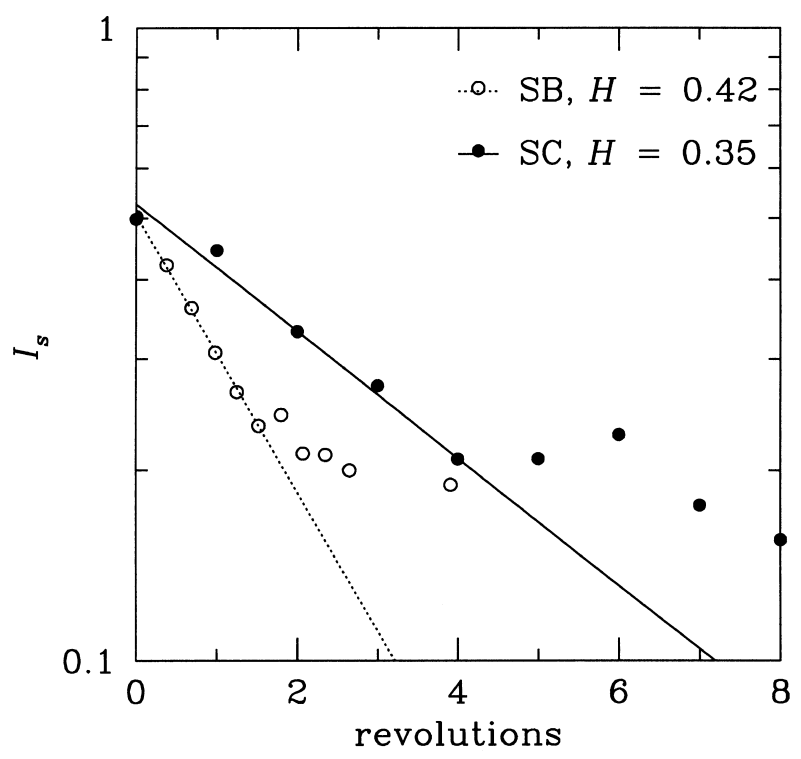

Fig. 4. Experimental intensity of segregation evolution for the experiments in Fig. 3. 


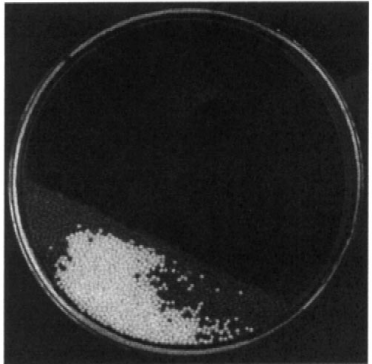

Experiment

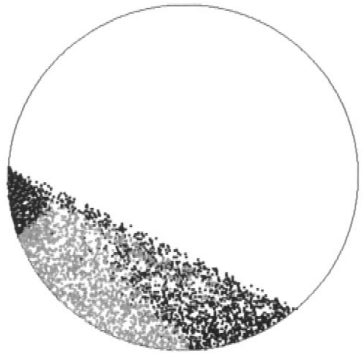

$\mathrm{D}=0.001$

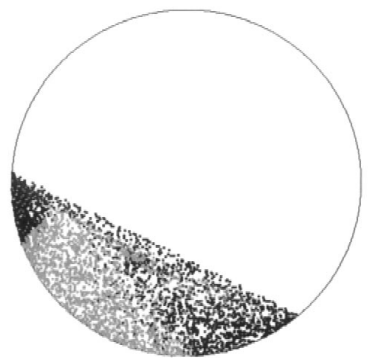

$\mathrm{D}=0.003$

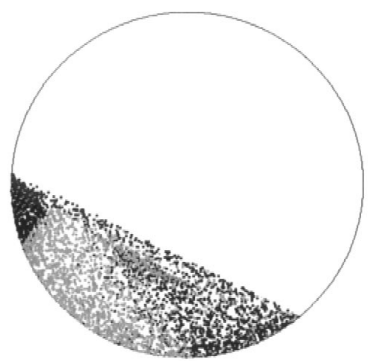

$\mathrm{D}=0.009$
Fig. 5. Qualitative comparison of experiment and computation with different values of the diffusivity for sugar balls.

A scaling expression by Savage [6], derived from PD Simulations for shear flow in a chute, has been proposed as:

$D=f(\nu) d^{2} \frac{\partial v_{x}}{\partial y}$

where $\nu$ is the void fraction, $f(\nu)$ is a decreasing function of void fraction (chosen to be $\mathscr{O}\left(10^{-2}\right)$ to fit our experiments), and $d$ is the particle diameter. Using this expression (and knowing $v_{x}$ ), values of the effective diffusivity

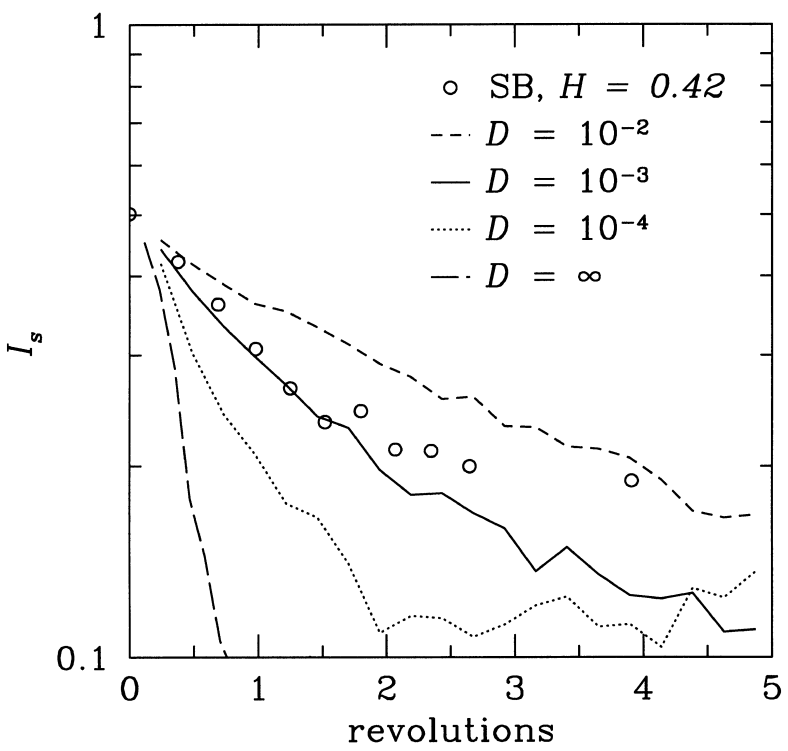

Fig. 6. Quantitative evaluation of the diffusivity for sugar balls. are calculated which are in good agreement with our experimentally estimated values. These results serve as an indirect experimental verification of Savage's [6] expression.
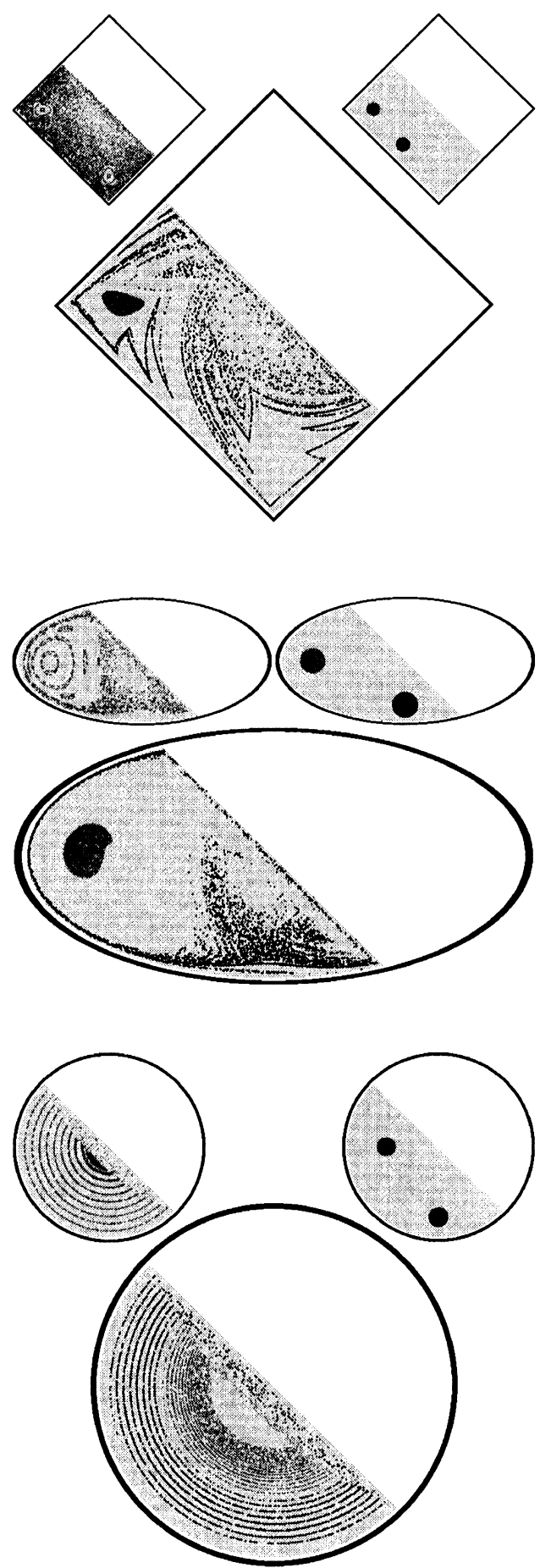

Fig. 7. Comparison of the mixing of tracer particles in a circular, elliptical, and square mixer (assuming no particle diffusion). The inset figure on the upper left shows the Poincare section, and the initial condition is shown in the upper right inset. Reprinted with permission from Khakhar et al., CHAOS 9(1), 1999, pp. 195-205. Copyright 1999 American Institute of Physics. 


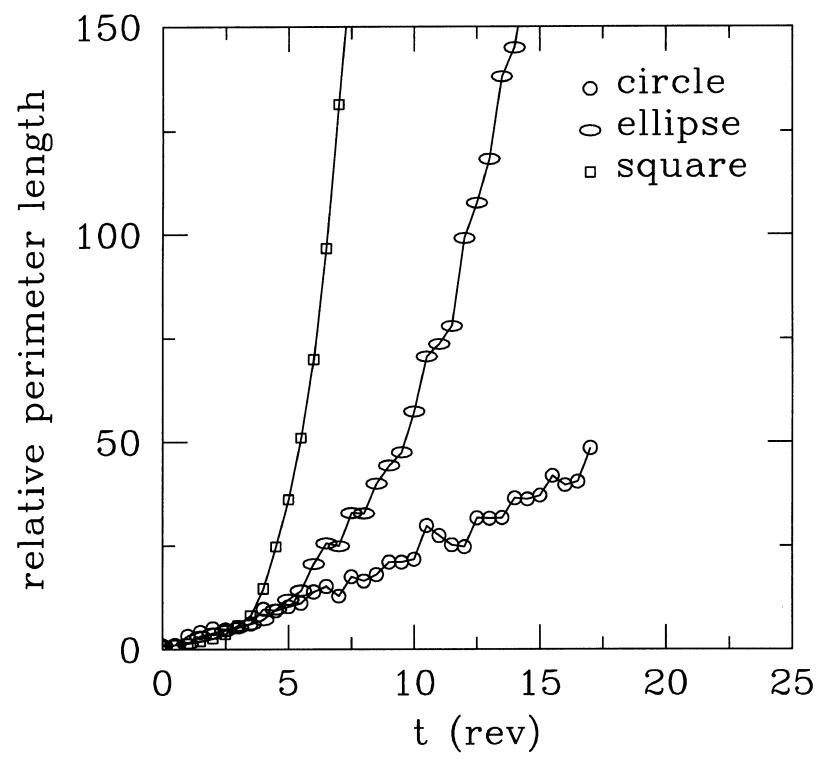

Fig. 8. Perimeter growth as mixing proceeds in different tumbler geometries. Exponential stretching is one of the signatures of chaos. Shown is the relative growth (scaled to its original length) of the perimeter of the blobs in Fig. 7. Note that the ellipse and square perimeter lengths grow exponentially, while the circle perimeter grows linearly. Reprinted with permission from Khakhar et al., CHAOS 9(1), 1999, pp. 195-205. Copyright 1999 American Institute of Physics.

\subsection{Mixing in non-circular cylinders - chaotic mixing}

The rolling regime is characterized by a steady flow. If one places a blob of material (from a continuum viewpoint) in the layer, one would see that upon rotation the blob would undergo stretching due to shear in the layer, while at the same time becoming blurred by particle collisions (collisional diffusion). The stretched and diffused blob eventually would leave the layer and would re-enter the bed, where it would undergo solid body rotation until the particles re-enter the layer and the process is repeated. When the cross-section of the rotating container is circular, the mean flow is time-independent, and the streamlines (lines tangent to mean velocity field) act as impenetrable barriers to convective mixing. This phenomenon is well understood; fluid mixing theory states that steady two dimensional flows are poor mixers [7]. Particles cross streamlines only by diffusive motion; mixing is consequently slow. Several studies of fluid flows show that time modulation of streamlines - such that there are intersections between streamlines obtained at different times - is generally sufficient to produce chaotic advection [8]. In a rotating tumbler, this simply happens when the cross-section is not circular [9].
This idea can be illustrated in terms of computations in circular (as a reference case) and elliptical containers and experiments and computations in square containers; in both non-circular cases the flow region - its free-surface length $2 L$, and its thickness $\delta_{\mathrm{o}}-$ becomes a function of the orientation of the container. This results in the streamline crossing condition necessary to produce chaos. In order to simulate the cases of the ellipse and the square, the model used is essentially the same as that of the previous section with the additional assumption that the layer geometry $\left(2 l(t)\right.$ and $\left.\delta_{0}(t)\right)$ varies with time sufficiently slowly that the flow is quasi-steady. Thus, the velocity field in the layer now becomes:

$v_{x}=\frac{\mathrm{d} x}{\mathrm{~d} t}=2 u\left(1+\frac{y}{\delta(x, t)}\right) l(t)$,

$v_{y}=\frac{\mathrm{d} y}{\mathrm{~d} t}=-x\left(\frac{y}{\delta(x, t)}\right)^{2}+S(t)$,

where quantities are now made dimensionless with the maximum half-length of the surface, $L, \delta(x, t)=\delta_{\text {om }}[1-$ $\left.\left[x / l^{2}(t)\right]\right] l(t)$, and $\delta_{\mathrm{om}}$ corresponds to the maximum layer thickness (obtained when the free-surface half-length is at its maximum, $L$ ).

Fig. 7 shows a comparison between the mixing of tracer particles in circular, elliptical, and square mixers obtained by means of computations. In order to isolate the effects of advection, the diffusivity in these simulations is set to zero. In the circular mixer (bottom) each initially circular blob (inset) is deformed into a filament; the length of the filament increases slowly with time. In contrast, in the case of the elliptical or square mixer (middle and top), markedly different behavior is seen. The initially circular blobs (inset) in the chaotic region are deformed into filaments which fold back upon themselves repeatedly. This indicates the presence of an underlying homoclinic structure which is characteristic of chaotic advection [7]. Similarly, one of the fingerprints of chaos is that material points which begin arbitrarily close to one another, separate exponentially. Fig. 8 shows the length of the blob perimeter for the circle, ellipse, and square normalized by its original length. Note that the perimeter of the blob in the circular mixer grows linearly, while in the elliptical and square mixers, the perimeters grow exponentially. On the other hand, the blobs in the regular regions of Fig. 7 deform only slowly over time. The differences in mixing for the geometries are highlighted in the computed Poincaré sections - stroboscopic maps of several test particle positions after every rotation of the mixer - shown as insets in Fig. 7. In the case of the circular mixer, the

Fig. 9. Mixing of tracer particles in a container with a square cross-section. Shown is a comparison of an experiment using colored glass beads (right) and a simulation using the model with $D=10^{-3}$ (left). The number of rotations for each image is listed in the corner. The blue blobs are placed initially at the approximate location of the hyperbolic points (see Poincaré sections in Fig. 7). Reprinted with permission from Khakhar et al., CHAOS 9(1), 1999, pp. 195-205. Copyright 1999 American Institute of Physics. 

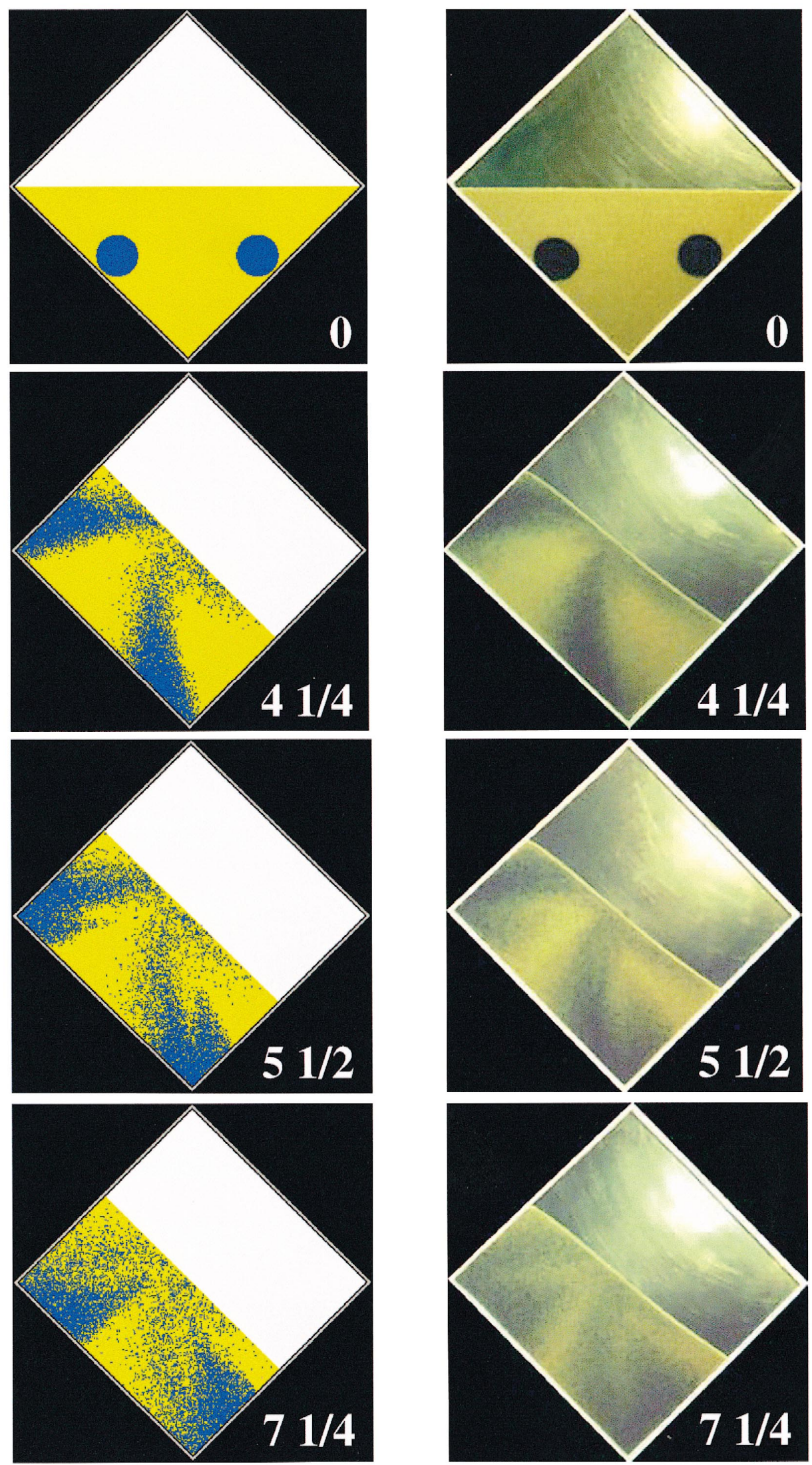

Computations

Experiments 
invariant surfaces of the Poincaré section coincide with the streamlines and there is no chaos. In the case of the elliptical or square mixer, however, regions of chaotic motion and regular motion are obtained.

Fig. 9 shows a comparison between computations and experiments in square containers. In this case, a fitted value of the collisional particle diffusion is incorporated into the model $\left(D=10^{-3}\right)$. Details of the experiments can be found in Ref. [9].

A visual examination of the experiments of Fig. 9 indicates that, as opposed to the case of fluids, where thin stretched and folded fluid striations can be resolved with little difficulty, diffusion plays a much more central role in granular mixing; however, diffusional effects in granular mixing are scale dependent. In the systems under consideration here, the Péclet number - the ratio of the characteristic advection time $(u / L)$ to the characteristic diffusion time $\left(\delta_{\mathrm{o}} / D\right)$ - is (in dimensional form):

$P e=\frac{\omega \delta_{\mathrm{o}} L}{2 D}$.

If we make Eq. (8) dimensionless and combine it with Eq. (5) for the diffusivity, after simplification we obtain:

$P e=\left(\frac{\kappa^{3}}{2 f(\nu)}\right)\left(\frac{L}{d}\right)^{2}$,

where $\kappa$ is the ratio of the maximum layer thickness, $\delta_{\mathrm{o}}$, to the maximum half-length, $L$. This ratio is seen to remain roughly constant for constant Froude number ( $\mathrm{Fr}$, the ratio of the rotational acceleration to the acceleration of gravity) over the range of experiments which we have conducted (see Fig. 10). Eq. (9) suggests that the effectiveness of diffusion decreases with increasing relative system

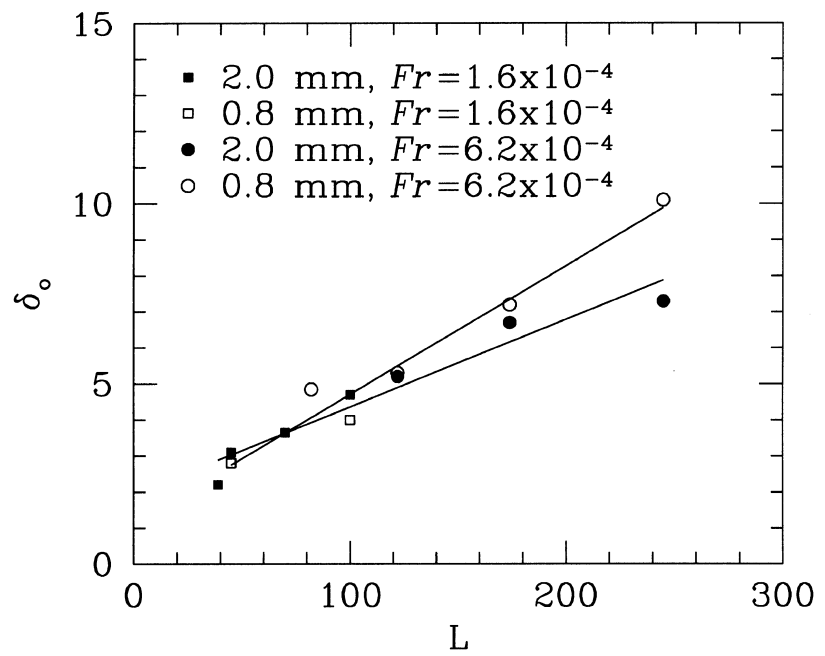

Fig. 10. Variation of the dimensionless mid-layer thickness, $\delta_{0}$, with the dimensionless length of the free surface, $L$, for different bead sizes, $d$, and Froude numbers, Fr. Circular and square symbols represent experiments run with 2 and $0.8 \mathrm{~mm}$ circular beads, respectively. Reprinted with permission from Khakhar et al., CHAOS 9(1), 1999, pp. 195-205. Copyright 1999 American Institute of Physics. size and would play a minimal roll in "large" systems. Also, for typical industrial systems, which would be considered large in this case, mixing in this regime is almost exclusively due to chaotic advection (provided that the mixer cross-section is not perfectly circular), a result which may have been overlooked if one were to rely on pilot-scale experimentation. In hindsight, existing processes undoubtedly already contain elements of chaotic advection (for example in V-blenders or double-cone blenders $[10,11]$ ); however, without explicit recognition of the underlying reasons for mixing successes and failures (global chaos vs. large-scale regular islands), optimization of such devices would be impossible.

\section{Particle Dynamics}

PD, a discrete method of simulation, is eminently suited to study one of the most difficult aspects of particulate flows, differing particle properties. This is especially useful in studying mixing flows, an area in which this technique has shown promise [11,12]. This method, however, is computationally intensive. While increasing computing power will certainly alleviate this difficulty somewhat, to date, this technique has found the majority of its success in the study of model systems designed to extract rules or heuristics useful for understanding more complex, macroscopic phenomena. In many cases of practical interest, insight gained from "experimentation" with model systems will allow these results to complement and perhaps improve other modes of simulation.

PD Simulations capture the macroscopic behavior of a particulate system via calculation of the trajectories of each of the individual particles within the mass; the time evolution of these trajectories then determines the global flow of the granular material. The particle trajectories are obtained via explicit solution of Newton's equations of motion for every particle [13]. The forces on the particles arise from interactions between particles (e.g., collisions or contacts) as well as interactions between the system and the particles (e.g., gravitational forces). For this work, the collisional forces are modeled after the work of Hertz and Mindlin [14]. A thorough description of the interaction laws can be found in Ref. [12]; therefore they will not be reviewed here.

\subsection{Cohesive systems}

The vast majority of research in particulate flows has been directed at the study of particles which exhibit negligible attractive forces between individual grains (i.e., freeflowing powders) (some recent exceptions include Refs. [15-19]). This is due, primarily, to the difficulty involved in incorporating complex particle interactions - which potentially vary with particle separation, velocity, size, or 
other particle properties - into a continuum or other model. In contrast, in a PD Simulation, additional particle interaction forces (such as surface adhesion [18], van der Waals forces [20], etc.) can be easily added.

Here, we focus our attention on moderately sized particles, $\sim 1 \mathrm{~mm}$, where forces attributable to interstitial liquid are the dominant cohesive interaction effect [21]. The phenomenon of liquid bridging between particles was first treated quantitatively by Fisher [22], and recently by Lian et al. [23]. Using these models, the cohesive force of a liquid bridge - accounting for the surface tension and pressure difference components - can be calculated as a function of particle separation. In addition to the capillary force, "wet" particles encounter a viscous force resisting motion, which can be derived from lubrication theory (see, for example Ref. [24]; a derivation specific to the case of particles is given in Ref. [25]). One final consideration is the surface roughness of the particles. Strictly speaking, perfectly smooth spheres would encounter a decreasing attractive force for increasing liquid content (while in the pendular regime), and viscous forces could become arbitrarily large for infinitely small separation distances. In practice, surface asperities limit the minimum separation distance to some constant value, $S_{\mathrm{o}}$. In this work, $S_{\mathrm{o}}$ $\sim 10^{-6} \mathrm{~m}$, a reasonable value for commercial grade particles. The algorithm used here is quite similar to that reported in Ref. [26] and will not be elaborated here.

A simple characterization measure, the granular Bond number $(B o)$, is suggested as a means of quantifying the effect of cohesion on the flow of the material. The granular Bond number is defined, generally, as the ratio of the attractive force to particle weight. Clearly, this definition can lead to at least three distinct quantities: a global $B o$, based on a suitable averaged attractive force and the average particle mass; a local $B o$, based on the instantaneous attractive force between a specific pair of particles and the weight of the heavier of the pair; and the average $B o$, computed as the mean of all of the local $B o$.
Based on a capillary force view of particle cohesion, the global granular Bond number can be defined as:

$B o=\frac{F_{\mathrm{c}}}{W}=\frac{2 \pi \sigma a}{\frac{4}{3} \pi a^{3} \rho g}=\frac{3 \sigma}{2 a^{2} \rho g}$,

where $F_{\mathrm{c}}$ is a simple model of the capillary force, $W$ is the particle weight, $a$ is the radius, $\rho$ is density, and $\sigma$ is the liquid surface tension.

The simulations in this section were carried out at rotation rates which were $10 \%$ of the critical value (when the angular acceleration is equal to the acceleration due to gravity). Fig. 11 shows a comparison of the mixing in tumblers at different $B o$ number. Qualitatively, it seems that the mixing rate at moderate $B o$ is faster than at small $B o$ (and then decreases again at large $B o$ ), a result which is somewhat contrary to intuition (similar results have recently been seen experimentally [27]). This suggests that, when some degree of moisture is present, there exists some optimum $B o$ for mixing within the rolling regime (a result with potential industrial significance). This idea is currently being explored further.

\subsection{Model flows - segregation due to density}

This section discusses surface segregation. While the procedure is somewhat general, the focus will be on density segregation in two model systems: chute flows and radial segregation. In Section 2, a LS model is proposed which adequately describes the mixing and flow in a continuously flowing tumbler. In this section, that model is expanded via development of a constitutive relationship for the segregation flux in cascading layers, primarily from model PD Simulations of chute flows. Consider equal sized particles of different densities flowing in a cascading layer. Higher density particles sink to lower levels in the layer, whereas lower density particles rise, resulting in segregation across the layer. The driving force for such segregation is the buoyant force experienced by the parti-

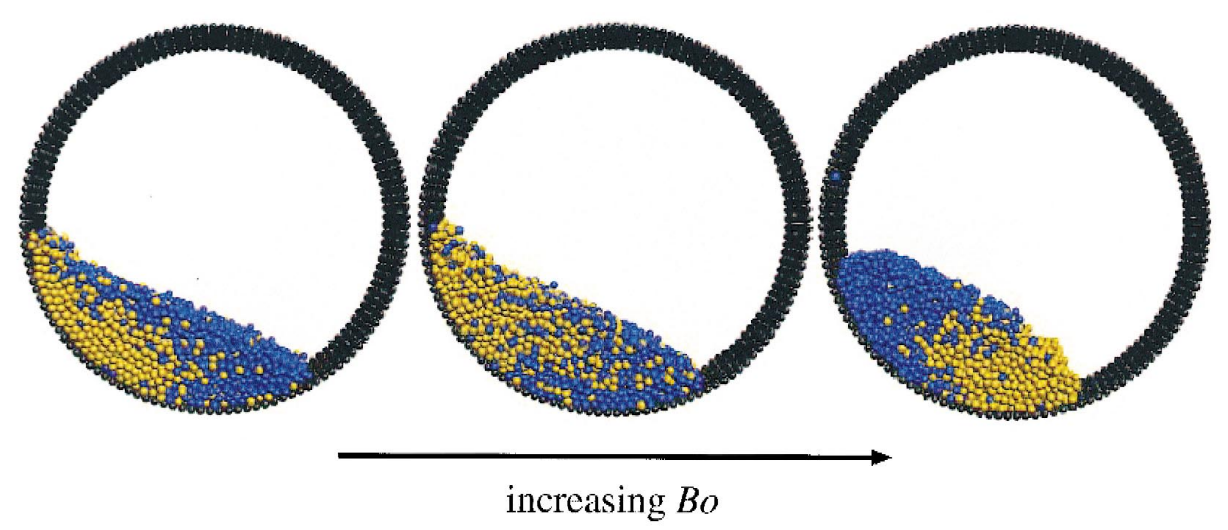

Fig. 11. A comparison of mixing at different Bo numbers for an initially segregated mixture (yellow particles began on the right and blue particles on the left) after two revolutions. 


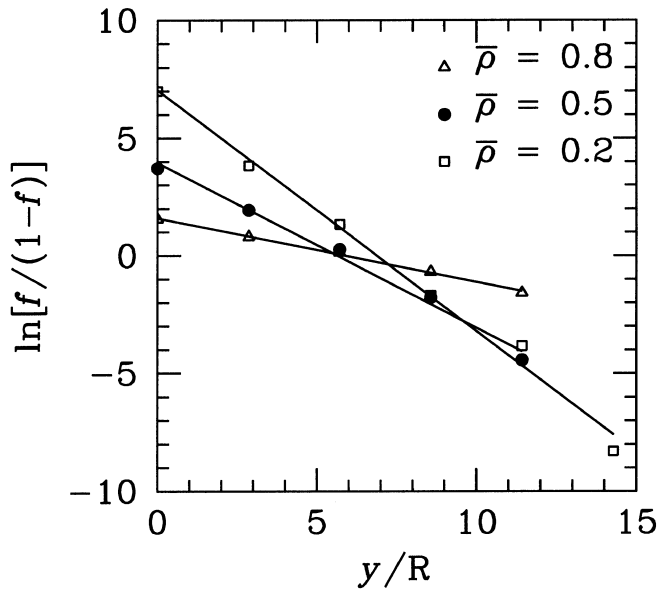

Fig. 12. Comparison of particle dynamics simulation of segregation in a chute flow with theory. Lines are fitted to data points for equilibrium segregation of different density ratios. For all three lines, a dimensionless segregation velocity of $\gamma=68$ is used.

cles; lighter particles may be considered to be immersed in an effective medium of higher density corresponding to the average density of the mixture, and heavier particles in a lower density effective medium. This idea forms the basis of a model for the segregation flux which is discussed below (a complete description of the derivation of the segregation flux model is given in Ref. [28]). The ultimate goal is to include into Eq. (3) a segregation velocity, $v_{\mathrm{s}}$, due to density differences, such that:

$v_{y}=\frac{\mathrm{d} y}{\mathrm{~d} t}=-x\left(\frac{y}{\delta(x)}\right)^{2}+S(t)+v_{\mathrm{s}}$,

is valid for density segregating systems.

As discussed in Section 2, interparticle collisions result in a diffusion flux which opposes the segregation. Hence, for sufficiently long layers and steady flow, a balance between segregation and diffusion fluxes results in an equilibrium concentration profile across the layer. The species balance equation in the layer at equilibrium is:

$0=\frac{\partial}{\partial y}\left(D \phi_{\mathrm{t}} \frac{\partial f}{\partial y}-J_{\mathrm{s}}\right)$,

where $f=\phi_{1} / \phi_{\mathrm{t}}, \phi_{1}$ is the volume fraction of the more dense particles, $\phi_{\mathrm{t}}=\left(\phi_{1}+\phi_{2}\right)$ is the total solids volume fraction, $D$ is the diffusivity, and $J_{\mathrm{s}}$ is the segregation flux of the more dense particles. For equal sized particles, as considered here, $f$ is simply the number fraction of the denser particles. Assuming the segregation velocity to be proportional to the buoyant force, the segregation velocity of the more dense particles is:

$v_{\mathrm{s}}=-\gamma D(1-f)$,

which yields a segregation flux, $J_{\mathrm{s}}$, of:

$J_{\mathrm{s}}=-\gamma D(1-f) f$.
At this point, the model contains a single parameter, the dimensionless segregation velocity, $\gamma$, which could be treated as a fitting parameter. Alternately, PD Simulations of chute flows can be used not only to validate the model for the segregation flux, but also to obtain an estimate of the dimensionless segregation velocity, $\gamma$. If one combines Eq. (14) with Eq. (12), one finds that the number fraction of the more dense particles, $f$, at equilibrium, should vary with height, $y$, as:

$\ln \frac{f}{1-f} \propto-\gamma y$.

A comparison of the simulation results for the number fraction, $f$, profiles to Eq. (15) is shown in Fig. 12. The agreement between the two is very good and there is, as predicted by the theory, a linear variation of $\ln (f /(1-f))$ with $y$. The value of $\gamma$ predicted by the PD Simulations, and subsequently used in the LS, is 68 (taken from Fig. 12).

Fig. 13 shows the results of a LS, using Eq. (11), of the equilibrium distribution of a segregating mixture of parti-

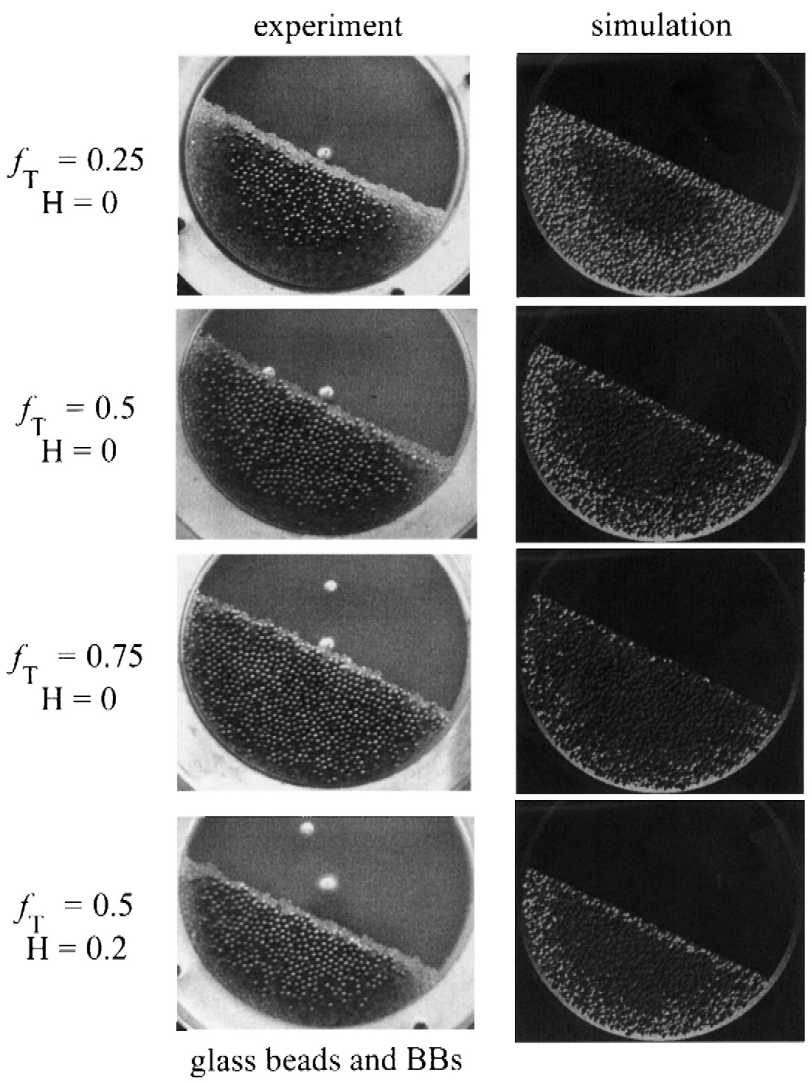

Fig. 13. Equilibrium distribution of beads obtained experimentally (left) and from LS (right) for differing filling, $H$, and total fraction of more dense particles, $f_{\mathrm{T}}$. The more dense particles are darker. Reprinted with permission from Khakhar et al., Fluids 9(12), 1997, pp. 1-14. Copyright 1997 American Institute of Physics. 

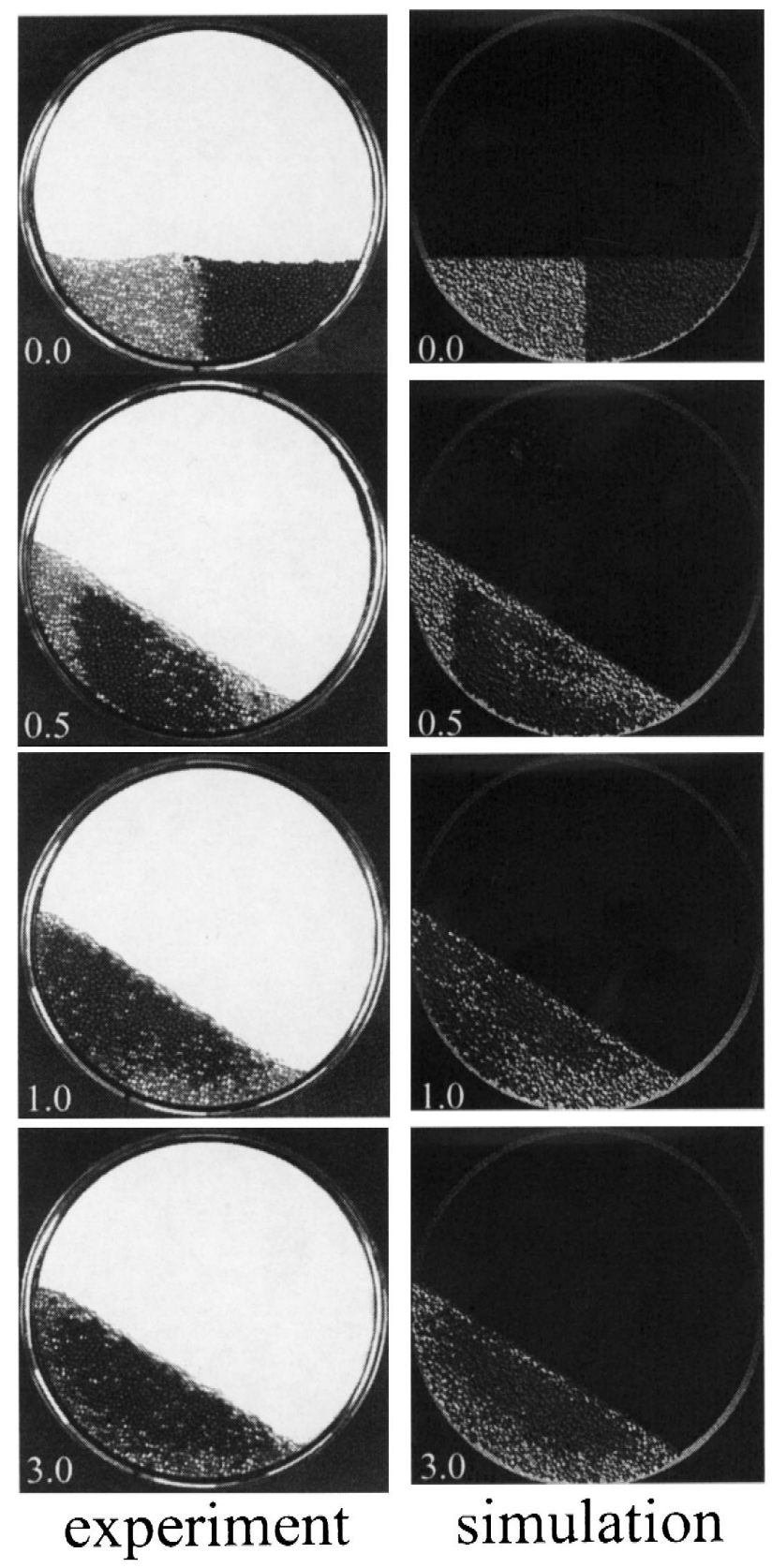

Fig. 14. Time evolution of the distribution of a mixture of particles of different density with rotation of the cylinder obtained experimentally (left) and from LS (right). Darker particles have higher density. Reprinted with permission from Khakhar et al., Physics of Fluids 9(12), 1997, pp. 1-14. Copyright 1997 American Institute of Physics.

cles in a rotated cylinder compared to experiment. There is good qualitative agreement both in the extent of segregation and the shape of the segregated core (a quantitative comparison of the results can be found in Ref. [28]).

In many cases, it is desirable to attempt to homogenize an initially segregated mixture of differing particles. In this case, the dynamics of mixing and segregation interact to give a complex evolution of the mixed state. Fig. 14 shows an example of such a process, in which an initially segre- gated state evolves to an equilibrium distribution. Experiments and the corresponding theoretical predictions are shown, and there is good agreement between the two. In both the simulation and the experiment, the system is better mixed at intermediate times as compared to the initial and final states. This becomes clearer if the mixed state at any time is quantified in terms of the intensity of segregation defined in Eq. (4). Fig. 15 shows the time evolution of the intensity of segregation for LS of two different extents of filling of the cylinder (the rate of advective mixing varies with filling, see Ref. [4]). For both fillings, the intensity of segregation decreases with time, indicating that mixing dominates over segregation at the initial stages of the process. At longer times, the change in $I_{\mathrm{s}}$ with time is different for different extents of filling. In the case of slow mixing (a lower value of $H$, corresponding to a more filled mixer), $I_{\mathrm{s}}$ monotonically decreases to the equilibrium value. In contrast, for fast mixing, $I_{\mathrm{s}}$ decreases to a minimum value and then increases to the same equilibrium value as the slow mixing case. Thus, the final mixed state is independent of the extent of filling, as found above; however, for fast mixing, there exists an optimal mixing time at which $I_{\mathrm{s}}$ is at its minimum. A minimum in the intensity of segregation has been observed in previous experimental studies of tumbling mixers [29]. While several empirical and semi-empirical approaches have been suggested to predict the typical behavior of segregating systems [30-33], the above theoretical and computational analysis is perhaps unique in having fundamental mechanisms as its basis and also for its lack of adjustable parameters.

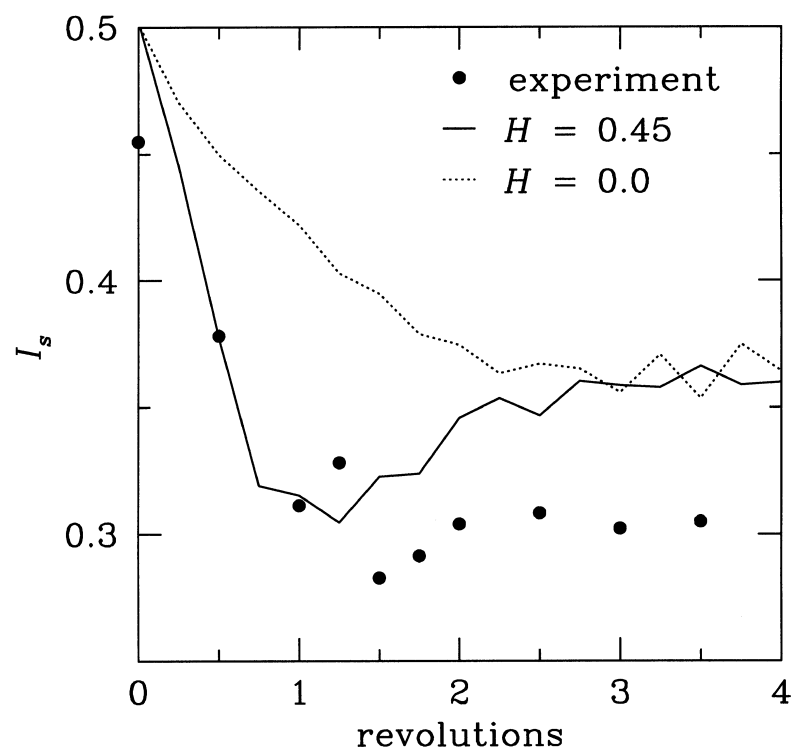

Fig. 15. Variation of the intensity of segregation with cylinder revolutions of an initially segregated mixture for different extents of filling of the cylinder. Curves are obtained from LS, points are from the experiment shown in Fig. 14. 


\section{Outlook}

Simulations of granular flows, and mixing flows in particular, provide a useful means of probing particulate behavior. In this paper, we focus on two very different, yet complementary, techniques for studying granular flow: PD and LS.

PD is versatile in that particle level interactions, including inelastic, frictional forces can be accounted for, such that segregation resulting from differences in particle properties can be explicitly studied. In addition, more complex particle interactions, such as cohesion, can also be included. However, large computational times are required, and simulation of industrial sized systems is currently not feasible.

LS are based on continuum models. Such models typically have fitting parameters to be determined by comparison to experiments, and, in general, a first principle approach starting from particle level interactions seems distant. The utility of such continuum models then depends on whether the fitting parameters are scale-independent. If this is the case, then experiments and PD Simulations for small systems can be used to design and optimize large scale systems.

The cases considered here (see Fig. 1) represent only a portion of the situations amenable to this type of attack. For example, segregation in non-circular containers [34,35] and in cohesive systems are currently being examined.

\section{Acknowledgement}

The authors would both like to acknowledge the Support of the Petroleum Research Fund as administered by the American Chemical Society.

\section{References}

[1] C.J. Broadbelt, J. Bridgwater, D.J. Parker, S.T. Keningley, P. Knight, Phenomenological study of a batch mixer using a positron camera, Powder Technol. 76 (1993) 317-325.

[2] M. Nakagawa, S.A. Altobelli, A. Caprihan, E. Fukushima, E.K. Jeong, Non-invasive measurements of granular flows by magnetic resonance imaging, J. Appl. Mech. 16 (1993) 256-270.

[3] M.S. Nikitidis, M.E. Hosseini-Ashrafi, U. Tüzün, N.M. Spyrou, Tomographic measurements of granular flows in gases and liquids, Kona 12 (1994) 53-66.

[4] D.V. Khakhar, J.J. McCarthy, T. Shinbrot, J.M. Ottino, Transverse flow and mixing of granular materials in a rotating cylinder, Phys. Fluids 9 (1997) 31-43.

[5] P.V. Danckwerts, The definition and measurement of some characteristics of mixtures, Appl. Sci. Res. A 3 (1952) 279-296.

[6] S.B. Savage, Disorder, diffusion and structure formation in granular flow, in: D. Bideau, A. Hansen (Eds.), Disorder and Granuar Media, Elsevier, Amsterdam, 1993, pp. 255-285.

[7] J.M. Ottino, The Kinematics of Mixing: Stretching, Chaos, and Transport, Cambridge Univ. Press, Cambridge, 1990.

[8] J.M. Ottino, F.J. Muzzio, M. Tjahjadi, J.G. Franjione, S.C. Jana, H.A. Kusch, Chaos, symmetry, and self-similarity: exploiting order and disorder in mixing processes, Science 257 (1992) 754-760.
[9] D.V. Khakhar, J.J. McCarthy, J. Gilchrist, J.M. Ottino, Chaotic advection of granular materials, CHAOS 9 (1999) 195-205.

[10] M. Poux, P. Fayolle, J. Bertrand, D. Bridoux, J. Bousquet, Powder mixing, Powder Technol. 68 (1991) 213-234.

[11] C. Wightman, P.R. Mort, F.J. Muzzio, R.E. Riman, R.K. Gleason, The structure of mixtures of particles generated by time-dependent flows, Powder Technol. 84 (1995) 231-240.

[12] J.J. McCarthy, J.M. Ottino, Particle dynamics simulation: a hybrid technique applied to granular mixing, Powder Technol. 97 (1998) 91-99.

[13] P.A. Cundall, O.D.L. Strack, A discrete numerical model for granular assemblies, Geotechnica 29 (1979) 47-65.

[14] K.L. Johnson, Contact Mechanics, Cambridge Univ. Press, Cambridge, 1987.

[15] D. Hornbaker, R. Albert, I. Albert, A.-L. Barabási, P. Schiffer, What keeps sandcastles standing, Nature 387 (1997) 765-767.

[16] R. Albert, I. Albert, D. Hornbaker, P. Schiffer, A.-L. Barabási, The angle of repose in wet and dry granular media, Phys. Rev. E 56 (1997) R6271-R6276.

[17] M. Abdel-Ghani, J.G. Petrie, J.P.K. Seville, R. Clift, Mechanical properties of cohesive particulate solids, Powder Technol. 65 (1991) $113-123$.

[18] C. Thornton, K.K. Yin, M.J. Adams, Numerical simulation of the impact fracture and fragmentation of agglomerates, J. Phys. D 29 (1996) 424-435.

[19] P. Pierrat, H.S. Caram, Tensile strength of wet granular materials, Powder Technol. 91 (1997) 83-93.

[20] K.Z.Y. Yen, T.K. Chaki, A dynamic simulation of particle rearrangement in powder packings with realistic interactions, J. Appl. Phys. 71 (1992) 3164-3173.

[21] H. Rumpf, Particle Technology, Chapman \& Hall, New York, 1975.

[22] R.A. Fisher, On the capillary forces in an ideal soil, J. Agric. Sci. 16 (1926) 491-505.

[23] G. Lian, C. Thornton, M.J. Adams, A theoretical study of liquid bridge forces between two rigid spherical bodies, J. Colloid Interface Sci. 161 (1993) 138-147.

[24] R.B. Bird, W.E. Stewart, E.N. Lightfoot, Transport Phenomena, Wiley, New York, 1960.

[25] M.J. Adams, B. Edmonson, Forces between particles in continuous and discrete liquid media, in: B.J. Briscoe, M.J. Adams (Eds.), Tribology in Particulate Technology, Adam Hilger, Philadelphia, 1987, pp. 154-172.

[26] G. Lian, C. Thornton, M.J. Adams, Discrete particle simulation of agglomerate impact coalescence, Chem. Eng. Sci. 53 (1998) 33813391.

[27] T. Shinbrot, A. Alexander, F.J. Muzzio, Spontaneous chaotic granular mixing, Nature 397 (1999) 675-678.

[28] D.V. Khakhar, J.J. McCarthy, J.M. Ottino, Radial segregation of granular materials in rotating cylinders, Phys. Fluids 9 (1997) 36003614.

[29] S.S. Weidenbaum, C.F. Bonilla, A fundamental study of the mixing of particulate solids, Chem. Eng. Prog. 51 (1955) 27-35.

[30] H.E. Rose, A suggested equation relating to the mixing of powders and its application to the study of the performance of certain types of machine, Trans. Inst. Chem. Eng. 37 (1959) 4-12.

[31] M. Alonso, M. Satoh, K. Miyanami, Optimum combination of size ratio, density ratio and concentration to minimize free surface segregation, Powder Technol. 68 (1991) 145-152.

[32] J. Boss, Mixing time of grain materials, Chem. Eng. Sci. 47 (1992) 4027-4035.

[33] J. Bridgwater, Fundamental powder mixing mechanisms, Powder Technol. 15 (1976) 215-231.

[34] K.M. Hill, D.V. Khakhar, J.F. Gilchrist, J.J. McCarthy, J.M. Ottino, Segregation-driven organization in chaotic granular flows, PNAS 96 (1999) 11701-11706.

[35] J.M. Ottino, D.V. Khakhar, Mixing and segregation of granular materials, Annual Review of Fluid Mechanics 32 (2000) 55-91. 